\title{
Limb reduction defects in Emilia Romagna, Italy: epidemiological and genetic study in 173109 consecutive births
}

\author{
Elisa Calzolari, Daniela Manservigi, Gian Paolo Garani, Guido Cocchi, Cinzia Magnani, \\ Mario Milan
}

\begin{abstract}
Epidemiological and genetic variables in limb reduction defects (LRD) were analysed during the years 1978 to 1987 in a case control study in Emilia Romagna, northern Italy. During the observation period, 83 neonates out of 173109 consecutive births had LRD (4.8 per 10 000). Cases were divided into five subgroups: transverse, intercalary, longitudinal, split, and multiple types of LRD. Of all cases, $64 \%$ were upper limb, $21 \%$ lower limb, and $15 \%$ both. Coexisting non-limb malformations were found in 10 cases (12\%), five with recognised syndromes and five with other associated defects. About $\mathbf{7 \cdot 2 \%}$ of first degree relatives had defects involving the skeletal system. In two cases the mother had the same type of LRD (a split). No recurrence among sibs was observed. Risk factors correlated with LRD were found to be low birth weight ( $2500 \mathrm{~g}$ or less), vaginal bleeding, and threatened abortion.
\end{abstract}

Limb reduction defects (LRD) are severe anomalies occurring in about one in every 2000 (five per 10000 ) newborn infants. ${ }^{1-4}$ Some of these anomalies are genetic in origin, resulting from dominant and recessive genes $^{56}$ and chromosomal abnormalities. ${ }^{7}$

Istituto di Genetica Medica, University of Ferrara, Via L Borsari 46, 44100 Ferrara, Italy.

E Calzolari, D Manservigi, M Milan

Istituto di Pediatria, University of Ferrara, Italy. G P Garani

Istituto di Puericultura, University of Bologna, Italy. G Cocchi

Istituto di Puericultura e Medicina Neonatale, University of Parma, Italy.

C Magnani

Correspondence to Dr Calzolari.

Received for publication 12 October 1989.

Revised version accepted for publication 12 January 1990.
In addition, uterine factors (amniotic bands and mechanical and constraint problems), vascular compression, thrombosis, embolisation, ${ }^{8}$ and maternal diseases and infections (diabetes ${ }^{9}$ and herpes ${ }^{10}$ ) have been shown to cause limb malformations. At present thalidomide ${ }^{11}$ is the only known drug that can produce severe LRD in humans and only during a very brief period of early organogenesis (22 to 36 days postconception). Warfarin and anticonvulsants may produce digital hypoplasia by causing prolonged vascular or nutritional deprivation during pregnancy. ${ }^{12}$ However, the aetiology of many LRD is still unknown, and it is possible that a certain percentage can be attributed to the statistical probability that problems will arise during the complicated embryonic processes. ${ }^{13}$ In other words, to reduce the frequency of malformations beyond a certain limit may prove impossible despite continuing studies of the aetiology of malformations and preventive measures.

From a clinical point of view, LRD can either be isolated or part of syndromes or multiple non-limb malformations.

The present study reviews infants reported to have LRD in the Emilia Romagna register (1978 to 1987). Assessments were made in terms of frequency, referral patterns, co-occurring malformations, and aetiological factors.

\section{Materials and methods}

THE REGISTER

Details of the Emilia Romagna register for congenital malformations have been published previously. ${ }^{14-16}$ In this study, data on infants with congenital anomalies (whether born alive or stillborn after the 28th week of gestation) were reported using a specially designed questionnaire filled out by the medical staff during a personal interview with the mother. Each of these registered mothers was paired with a control mother whose delivery immediately preceded hers in the maternity unit. Diagnoses were written out rather than being given code numbers.

Descriptions of the malformations were supplemented with drawings, photographs, $x$ rays, and 
necropsy reports. All information was transmitted to the coordination centre, where it was reviewed by a trained clinician. If the information was incomplete, the missing data were requested from the reporting hospital.

EUROCAT guidelines ${ }^{17}$ were used to describe LRD.

\section{CLASSIFICATION OF LRD}

Limb reduction defects were defined as the absence or severe hypoplasia of skeletal structures of the limb. Excluded from the analysis were cases of brachydactyly and sirenomelia. The recorded cases were classified according to Temtamy and McKusick ${ }^{18}$ and EUROCAT guidelines. ${ }^{17}$

The following morphological types were differentiated (table 1).

(1) Terminal transverse: absence of the distal structures of the limb with the proximal structures more or less normal. Ectrodactyly was defined as partial or total absence of distal segments of the hand. The malformation can involve phalanges only (aphalangia), digits (adactylia), or the full hand (acheiria). All are considered to be various degrees of the same malformation.

(2) Intercalary: absence or severe hypoplasia of proximal (humerus and femur) and distal (radius and ulna or tibia and fibula) parts of the limb with normal or malformed hands and feet. The presence of distal skeletal structures differentiates intercalary defects from terminal transverse defects. The term phocomelia was avoided in this study because it has various meanings in published reports.

(3) Longitudinal: absence or severe hypoplasia of the lateral part of the limb. Two main subtypes were considered: preaxial (thumb, first metacarpal, radius; or hallux, first metatarsal, tibia) and postaxial (fifth finger, fifth metacarpal, ulna; or fifth toe, fifth metatarsal, fibula).

(4) Split hand/foot: paraxial or axial longitudinal terminal deficiency of rays, usually associated with syndactyly. Typical and atypical categories are recognised. The typical split category has two anatomical variants: lobster claw (absence of the central ray) and monodactyly (deficiency of radial rays with no clefts). The anatomical classification has no genetic significance because either type may occur in the same family or in different limbs of the same person. Generally, the atypical category is sporadic, affects only one hand (central rays are absent), does not involve the feet, and has hypoplastic digits on the axial portion of the hand.

(5) Multiple types of limb reduction: more than one type of LRD according to the definitions given above. The femur-fibula-ulna (FFU) complex was included in this category.

The cases in the study were divided into two major groups: (1) infants with only limbs affected (one or more); and (2) infants with coexisting non-limb malformations. In this group 'syndromes' and 'associations' were taken into consideration.

\section{CODES OF LRD}

The standard International Classification of Diseases (ICD) ${ }^{19}$ code was not detailed enough to allow classification of specific malformations and thus was inadequate for surveillance purposes. Therefore, we formulated a special numerical code, based on the morphological types listed above and in table 1, to classify each LRD more specifically.

Table 1 Classification of LRD identified in the Emilia Romagna register (1978 to 1987).

\begin{tabular}{|c|c|}
\hline Defect & Definition \\
\hline Terminal transverse & $\begin{array}{l}\text { Absence of the distal structures of the limb. The following types are considered: } \\
\text { Ectrodactyly: total or partial absence of phalanges (aphalangia), digits (adactylia), or full } \\
\text { hand (acheiria). } \\
\text { Amelia: total absence of extremities. } \\
\text { Hemimelia: total absence of forearm and hand or of foreleg and foot. } \\
\text { Apodia: absence of foot. }\end{array}$ \\
\hline Intercalary & $\begin{array}{l}\text { Absence or severe hypoplasia of proximal (radius and ulna or tibia and fibula) part of the limb } \\
\text { with hands and feet normal or malformed. The presence of distal skeletal structures } \\
\text { differentiates intercalary defects from terminal transverse. }\end{array}$ \\
\hline Longitudinal & $\begin{array}{l}\text { Absence or severe hypoplasia of lateral part of the limb: } \\
\text { Preaxial (thumb, first metacarpal, radius; or hallux, first metatarsal, tibia). } \\
\text { Postaxial (fifth finger, fifth metacarpal, ulna; or fifth toe, fifth metatarsal, fibula). }\end{array}$ \\
\hline Split hand/foot & $\begin{array}{l}\text { Paraxial or axial longitudinal terminal deficiency of rays, usually associated with syndactyly. } \\
\text { Two main anatomical varieties of typical split are: } \\
\text { Lobster claw (absence of the central ray). } \\
\text { Monodactyly (deficiency of radial rays with no clefts). } \\
\text { Atypical split resembles typical split type } 1 \text {. }\end{array}$ \\
\hline Multiple & More than one type of limb reduction (eg FFU complex). \\
\hline
\end{tabular}

bsence of the distal structures of the limb. The following types are considered:

Ectrodactyly: total or partial absence of phalanges (aphalangia), digits (adactylia), or full hand (acheiria).

Hemimelia: total absence of forearm and hand or of foreleg and foot. with hands and feet normal or malformed. The presence of distal skeletal structures

bsence or severe hypoplasia of lateral part of the limb:

Preaxial (thumb, first metacarpal, radius; or hallux, first metatarsal, tibia)

Paraxial or axial longitudinal terminal deficiency of rays, usually associated with syndactyly. wo main anatomical varieties of typical split are:

Lobster claw (absence of the central ray).

typical split resembles typical split type 1 . 
FACTORS ASSOCIATED WITH LRD

To test for an association between LRD and various epidemiological variables, syndromes with recognised Mendelian inheritance were excluded from the study.

Of the variables included on the registration form, the following were studied: infant's sex, parental age at delivery, history of selected maternal diseases, threatened abortion, maternal exposure to drugs and smoking during pregnancy, and other maternal factors including parity, previous abortions, stillbirths, and malformed infants.

The presence of LRD or other malformations in parents or sibs was recorded during interviews with the mothers.

\section{STATISTICAL ANAL YSIS}

Statistical tests included the $\chi^{2}$ test for the presence or

Table 2 Coexisting non-limb malformations: recognised syndromes or associations with LRD.

Fanconi: bilateral radial aplasia + bilateral hypoplasia of first metacarpal and first finger.

TAR: bilateral radial aplasia + thrombocytopenia + unspecified cardiac defect.

ADAM: ring encircling two fingers of the right hand and two toes of the left foot + limb constriction of the left leg with partial amputation of the foot.

Moebius: terminal transverse bilateral arms (acheiria bilateral) + terminal transverse unilateral leg + facial paralysis + micrognathia.

VATER: intercalary unilateral limb defect + atresia of oesophagus + ventricular septal defect + urinary tract anomalies.

Table 3 Coexisting non-limb malformations: other associated defects (each association occurred once).

Proximal intercalary defect unilateral leg + transposition of great vessels.

Preaxial longitudinal defect (absence of first metacarpal) + atresia of auricle + dextroversion.

Terminal transverse unilateral leg + spina bifida + gastroschisis + ambiguous genitalia.

Terminal transverse unilateral (amelia) + encephalocele + gastroschisis.

Terminal transverse unilateral (apodia) + atresia of rectum.

Table 4 Distribution of types of $L R D$ in 82 reported cases. absence of the characteristics under study in index patients and controls and the $t$ test for comparison of means. For detection of time clusters, we used the observed/expected ratio $^{20}$ and the cumulative sum techniques. ${ }^{21}$

\section{Results}

CHARACTERISTICS OF THE POPULATION UNDER STUDY

During the study period (1978 to 1987), 83 cases of LRD were detected among 173109 births in the Emilia Romagna region, giving a prevalence of 4.8 per 10000 .

There were $73(88 \%)$ isolated cases (one or more limbs affected) and $10(12 \%)$ with coexisting non-limb malformations, five $(6 \%)$ with recognised syndromes or associations (table 2 ) and five $(6 \%)$ with other associated defects (table 3).

A case of sirenomelia was excluded, and the remaining 82 cases were divided into six groups, according to the definitions described above (table 4).

Terminal transverse defects were the most common, occurring in 2.6 cases per 10000 . Other defects and their prevalences per 10000 included: split hand/foot 0.58 , longitudinal preaxial 0.52 , intercalary 0.46 , longitudinal postaxial 0.35 , and multiple LRD 0.23 (fig 1).

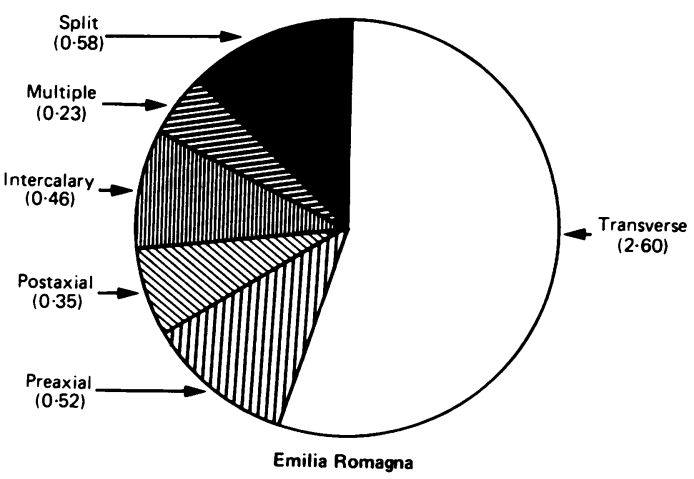

Figure 1 Rates of LRD per 10000 births identified in the register (1978 to 1987). 


\section{MALFORMATION FREQUENCY}

To study trends over time, as well as space and time clusters, all cases of LRD, including those associated with other defects, were considered. No significant trend was detected (analysis computed for successive and constant sets of 10000 births by De Wals and Lechat $\left.{ }^{22}\right)$. No marked intraregional differences for prevalence of LRD were noted $\left(\chi^{2}=8 \cdot 76\right.$, df $\left.7, \mathrm{NS}\right)$.

No time clusters were found with the cumulative sum technique.

\section{FACTORS ASSOCIATED WITH LRD \\ Cytogenetics}

Karyotypes were obtained in 15 of 83 cases; all were normal.

\section{Consanguinity and family data}

There was no instance of parental consanguinity among the affected cases or controls. In the affected group, six first degree relatives (about 7\%) had a musculoskeletal anomaly (two cases of congenital hip dislocation, two of polydactyly, two of split hand). In the control group, four first degree relatives had congenital hip dislocation.

\section{Environmental factors}

Of the several factors considered, only threatened abortion (bleeding or pain or both), treatment for abortion, and birth weight of $2500 \mathrm{~g}$ or less were related to LRD. Threatened abortion was diagnosed in $21.6 \%(16 / 74)$ of affected pregnancies as compared to $6.0 \%(5 / 83)$ of controls $\left(\chi^{2}=6.42\right.$, df $\left.1, p<0.05\right)$.

Treatment for threatened abortion or history of previous abortion or both was given in $31.1 \%$ of index pregnancies $(23 / 74)$ and $9 \cdot 6 \%$ of controls $(8 / 83)$ $\left(\chi^{2}=10 \cdot 27\right.$, df $\left.1, \mathrm{p}<0.001\right)$.

The mean birth weight for the index cases was $2882 \mathrm{~g}$ as compared to $3363 \mathrm{~g}$ for the control group. The difference is significant $(t=5.09, \mathrm{p}<0.001)$. In particular, birth weights of $2500 \mathrm{~g}$ or less were found in $21.5 \%(17 / 79)$ of the LRD cases and in $2 \cdot 5 \%(2 / 80)$ of controls $\left(\chi^{2}=11.92\right.$, df $\left.1, p<0.001\right)$. These findings are in keeping with those reported by Aro et al,,$^{23}$ Polednak and Janerich, ${ }^{25}$ and Kallen. ${ }^{26}$

\section{Discussion}

The purpose of this study was to analyse limb reduction defects as reported in the Emilia Romagna register (1978 to 1987). The prevalence of LRD in this region $(4 \cdot 8 / 10000)$ was in keeping with that observed elsewhere in Italy ${ }^{27}$ and in other countries. ${ }^{422} 28$ The rates of types of LRD (fig 2) indicate that, in accordance with published reports, terminal

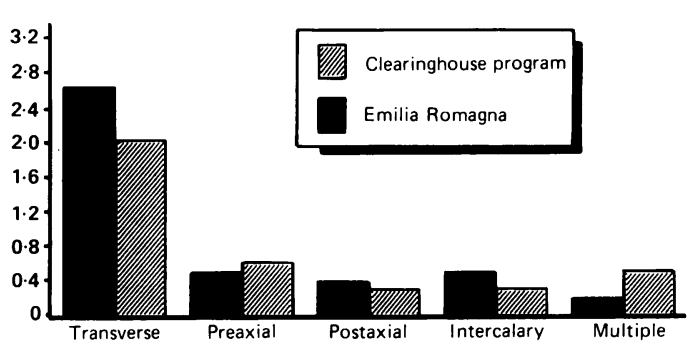

Figure 2 Rates of LRD per 10000 births by type of defect, Emilia Romagna and Clearinghouse data.

transverse defects are the most common $(2 \cdot 6 / 10000)$, and are followed in descending order by longitudinal preaxial $(0.52)$, intercalary $(0.46)$, and longitudinal postaxial $(0 \cdot 35)$.

The prevalence of split hand/foot $(0.58 / 10000)$ in our study did not differ greatly from that reported in Hungary $(0 \cdot 4){ }^{1}$

The frequency of LRD with coexisting non-limb malformations was $12 \%$ (five cases with recognised syndromes and five with other associated defects), while the percentage of associated anomalies was $30 \%$ in a Swedish study ${ }^{2}$ and $53 \%$ in a Canadian study. ${ }^{4}$ This difference is because in our study the cases of LRD coexisting with other malformations of the musculoskeletal system were not considered as associated defects (for example, polydactyly or triphalangeal thumb described in association with split malformation, or talipes with absence of fibula). In addition, the simultaneous occurrence of the same type of defect (for example, terminal transverse in upper and lower limb) was not considered to be an associated defect.

No intraregional variations in prevalence were observed, and no time clusters were identified with the observed/expected ratio or cumulative sum techniques. Special codes were used for surveillance purposes.

No recurrences among sibs were observed. In two of 10 cases of split, a mother and child were affected, which may be attributable to autosomal dominant inheritance.

Among the variables considered in the present study, threatened abortion, treatment for abortion (for example, hormones), and birth weight of $2500 \mathrm{~g}$ or less were significantly related to LRD. These are the only three variables that were found to be consistently correlated with LRD in previous reports. ${ }^{23-}$ 25

Vaginal bleeding and threatened abortion can be considered indicators for impaired embryogenesis, but are not specifically associated with LRD.

We are grateful to Giovanna Azzini for technical assistance and to Patricia Ennis for her helpful 
comments concerning manuscript preparation. The authors are also indebted to the many physicians, midwives, nurses, and registry clerks in the participating centres for their assistance in data collection. This work was supported by grant MPI $40 \% 1981$, 1982, 1983, 1984, 1985 and the Fondo Regionale Progetto Obiettivo no 3553/6513.

1 Bod M, Czeizel A, Lenz W. Incidence at birth of different types of limb reduction abnormalities in Hungary 1975-1977. Hum Genet 1983;65:27-33.

2 Kallen B, Rahmani TMZ, Winberg J. Infants with congenital limb reduction registered in the Swedish register of congenital malformations. Teratology 1984;29:73-85.

3 EUROCAT Working Group. Surveillance of congenital anomalies, years 1980-1986. Brussels: Department of Epidemiology, Catholic University of Louvain, 1989

4 Froster-Iskenius UG, Baird PA. Limb reduction defects in over one million consecutive livebirths. Teratology 1989;39:127-35.

5 Pilarski RT, Pauli RM, Engberg WD. Hand reduction malformations: genetic and syndromic analysis. $\mathcal{F}$ Pediatr 1985;5:274-80.

6 Pauli RM, Lebovitz RM, Meyer RD. Familial recurrence of terminal transverse defects of the arm. Clin Genet 1985;27: 555-63.

7 Haspeslagh M, Fryns JP, Moerman P. Severe limb malformations in 4p deletion. Clin Genet 1984;25:253-6.

8 Bouwes-Bavinck JN, Weaver DD. Subclavian artery supply disruption sequence: hypothesis of a vascular etiology for Poland, Klippel-Feil and Moebius anomalies. Am $\mathcal{F}$ Med Genet 1986;23:902-19.

9 Johnson JP, Carey JC, Gooch WM III, Peterson J, Beattie JF. Femoral hypoplasia-unusual facies syndrome in infants of diabetic mothers. $\mathcal{F}$ Pediatr 1983;102:866-72.

10 Stagno S, Whitley RJ. Herpes virus infections of pregnancy. N Engl f Med 1985;313:1327-9.

11 Brent RL, Holms LB. Clinical and basic science lessons from the thalidomide tragedy: what have we learned about the causes of limb defects? Teratology 1988;38:241-51.

12 Goodman RM, Gorlin RJ. The malformed infant and child. New York: Oxford University Press, 1983.
13 Kurnit DM, Layton WN. Genetics, chance and morphogenesis. Am f Hum Genet 1987;41:979-95.

14 Calzolari E, Contiero MR, Roncarati E, Mattiuz PL, Volpato S. Aetiological factors in hypospadias. F Med Genet 1986;23: 333-7.

15 Calzolari E, Cavazzuti GB, Cocchi G, et al. Congenital malformations in 100000 consecutive births in Emilia Romagna region, northern Italy: comparison with the EUROCAT data. Eur $\mathcal{F}$ Epidemiol 1987;3:423-30.

16 Calzolari E, Milan M, Cavazzuti GB, et al. Epidemiological and genetic study of $\mathbf{2 0 0}$ cases of oral clefts in the Emilia Romagna region of northern Italy. Teratology 1988;38:559-64

17 Stoll C, Mastroiacovo PP, DeWals P, Weatherall JAC, eds. EUROCAT guide for the description and classification of congenital limb defects. Brussels: Department of Epidemiology, Catholic University of Louvain. 1986

18 Temtamy SA, McKusick VA. The genetics of hand malformations. New York: Alan R Liss, 1976:53-71.

19 British Paediatric Association Classification of Disease. A paediatric supplement compatible with the ninth revision of the WHO international classification of diseases, 1977.

20 Weatherall JAC, Haskey JC. A test for detection of clustering over time. Am F Epidemiol 1980;111:367-72.

21 Barbujani G. A review of statistical methods for continuous monitoring of malformation frequencies. Eur $\mathcal{F}$ Epidemiol 1987;3:67-77.

22 De Wals P, Lechat MF. Surveillance of congenital anomalies, years 1980-1984. EUROCAT Report 2. Brussels: Department of Epidemiology, Catholic University of Louvain, 1987.

23 Aro T, Heinonen OP, Saxen L. Risk indicators of reduction limb defects, 7 Epidemiol Community Health 1983;37:50-6.

24 Aro T, Haapakoski J, Heinonen OP. A multivariate analysis of the risk indicators of reduction limb defects. Int $\mathcal{f}$ Epidemio 1984;13:459-64.

25 Polednak AP, Janerich DT. Maternal factors in congenital limbreduction defects. Teratology 1985;32:41-50.

26 Kallen B. A prospective study of some epidemiological factors in limb reduction defects in Sweden. Int 7 Epidemiol Community Health 1989;43:86-91

27 Malformazioni congenite (anno 1983). Bollettino Epidemiologico Nazionale, 1983;83/34:355.

28 International clearinghouse for birth defects monitoring systems. Annual report 1986. 